\title{
Correction to: A Systematic Review of Community Health Workers' Role in Occupational Safety and Health Research
}

\author{
Jennifer E. Swanberg ${ }^{1,2}$ (1) Helen M. Nichols ${ }^{1}$. Jessica M. Clouser ${ }^{3} \cdot$ Pietra Check $^{4} \cdot$ Lori Edwards $^{5} \cdot$ Ashley M. Bush $^{6}$. \\ Yancy Padilla ${ }^{1} \cdot$ Gail Betz ${ }^{7}$
}

Published online: 3 April 2018

○) Springer Science+Business Media, LLC, part of Springer Nature 2018

\section{Correction to: Journal of Immigrant and Minority Health https://doi.org/10.1007/s10903-018-0711-z}

The original version of this article unfortunately contained a mistake in the affiliation of co-author Ashley M. Bush. The correct affiliation is Kentucky Injury Prevention and Research Center, College of Public Health, University of Kentucky, Lexington, KY, USA.

The original article can be found online at https://doi.org/10.1007/ s10903-018-0711-z.

Jennifer E. Swanberg

jswanberg@ssw.umaryland.edu

1 University of Maryland School of Social Work, Baltimore, MD, USA

2 Department of Epidemiology and Public Health, University of Maryland School of Medicine, Baltimore, MD, USA

3 Center for Health Services Research, College of Medicine, University of Kentucky, Lexington, KY, USA

4 Department of Public Health and Community Medicine, Tufts University School of Medicine, Boston, MA, USA

5 University of Maryland School of Nursing, Baltimore, MD, USA

6 Kentucky Injury Prevention and Research Center, College of Public Health, University of Kentucky, Lexington, KY, USA

7 Health Sciences and Human Services Library, University of Maryland, Baltimore, Baltimore, MD, USA 